\title{
Le tecniche e le soluzioni utilizzate nella detersione delle ulcere: una revisione della letteratura
}

\author{
Elsa Vitale, ${ }^{1}$ Lucia Rosa De Angelis, ${ }^{2}$ Francesco Germini ${ }^{3}$ \\ ${ }^{1}$ Department of Mental Health ASL Bari; ${ }^{2}$ Vascular Surgery ward, Di Venere hospital ASL Bari; ${ }^{3}$ Manager of Health Professions, \\ ASL Bari, Italy
}

\section{RIASSUNTO}

L'ulcera è la perdita di continuità dell'epitelio, dell'epidermide o della mucosa causata dall'espulsione del tessuto necrotico infiammato. È l'espressione di processi degenerativi provocati da fenomeni infiammatori, infettivi, da disturbi circolatori o da danneggiamento tissutale per cause chimiche e fisiche. Il trattamento di una lesione cutanea cronica deve essere multidisciplinare e richiedere la collaborazione di numerosi specialisti, del personale infermieristico, del paziente e/o del nucleo familiare. Lo scopo della revisione è quello di verificare gli effetti della detersione sulle ferite considerando sia gli approcci adottati, sia le soluzioni utilizzate, valutando i relativi tassi di guarigione e di infezione delle ulcere. La ricerca della letteratura è stata eseguita nelle banche dati di Pubmed e Cochrane Library. Sono stati considerati studi clinici randomizzati e revisioni di letteratura dal 2003 al 2018.276 lavori scientifici sono stati individuati. Di questi, sono 15 studi sono stati considerati potenzialmente eleggibili. Successivamente alla lettura completa di ciascun lavoro sono stati inclusi infine 6 articoli. La gestione delle ulcere coinvolge molte figure professionali esperte in wound care che dovrebbero collaborare tra loro per guarire le ferite. Specificamente la detersione viene considerata un intervento di routine che viene effettuato con soluzione fisiologica sterile attraverso il metodo del tamponamento, questo perché la letteratura non fornisce ricerche soddisfacenti nell'ambito dell'utilizzo dell'acqua di rubinetto.

\section{INTRODUZIONE}

Recentemente un panel di esperti ha raggiunto il consenso sulla definizione ulcera come: un'alterazione della normale struttura anatomica e funzionale dei tessuti, determinata da processi patologici endogeni ed esogeni rispetto agli organi interessati, che non evolve secondo il normale processo ripartivo. ${ }^{1}$ L'impatto che l'ulcera determina sulla qualità di vita del paziente è ampiamente documentata: il dolore, la frustrazione che l'ulcera non potrebbe guarire, le recidive, i problemi nella mobilità,

Corrispondenza: Vitale Elsa, ASL Bari, Centre of Mental Health, via $X$ marzo n. 43, Modugno, Bari, Italia.

Tel.: +393339910154 .

E-mail: vitaleelsa@libero.it,

Conflitti di interesse: Gli autori dichiarano l'assenza di confitti di interesse.

Parole chiave: Abrasione; Ferita; Ulcera; Lacerazione; Pulizia.

Ricevuto per la pubblicazione: 17 maggio 2019.

Accettato per la pubblicazione: 6 maggio 2020 .

This work is licensed under a Creative Commons Attribution NonCommercial 4.0 License (CC BY-NC 4.0).

${ }^{\circ}$ Copyright: the Author(s), 2020

Licensee PAGEPress, Italy

Italian Journal of Wound Care 2020; 4(1):15-22

doi:10.4081/ijwc.2020.51 l'isolamento sociale, la mancata aderenza al trattamento terapeutico sono tutte problematiche attive in questa categoria di pazienti, considerati cronici. Nella pratica clinica la documentazione dell'ulcera include: la raccolta della storia clinica, l'osservazione della sede, della profondità e delle dimensioni, la descrizione dell'aspetto del letto della ferita e della cute circostante, un'analisi dell'essudato e la segnalazione della presenza di dolore.

La sfida che devono affrontare i professionisti sanitari consiste nell'istituire strategie terapeutiche efficaci in modo tempestivo ed economicamente vantaggioso, con l'obiettivo di ridurre la complessità della ferita, trattare i sintomi del paziente, rispondere alle sue aspettative e, se possibile, raggiungere la piena guarigione della ferita. Nell'ambito del wound care l'infermiere gestisce molteplici aspetti quali: la gestione della terapia, la medicazione, l'educazione, l'ottimizzazione della compliance alla terapia, la gestione del dolore., ${ }^{2,3}$

Tra gli argomenti di maggior rilievo nella pratica infermieristica del wound care si annovera la detersione delle ferite. Quest'ultima è considerata come il primo momento della cura delle ferite, spesso identificata come un'azione rituale che, tuttavia se in appropriatamente eseguita può causare ritardi nella guarigione.

In letteratura si trovano differenti concezioni teoriche di detersione. Ad esempio nell'approccio completo olistico al paziente affetto da ulcere: esiste il paziente con una ferita, non una ferita con il paziente. ${ }^{4}$

La letteratura definisce la detersione come l'applicazione di un liquido sulla ferita, solitamente prima 
di applicare una medicazione allo scopo di facilitare la rimozione di essudato, detriti solubili e contaminati, ma non comprende l'uso di medicazioni o sbrigliamento meccanico. ${ }^{1,6}$

La realtà dei fatti è che in molti contesti clinici la detersione viene eseguita più per abitudine che per necessità. Se la detersione avviene in modo automatico, ciò implica che i professionisti della salute non si interrogano su cosa significhi detergere una ferita o un'ulcera, né riflettono su cosa intendono ottenere detergendole.

La detersione rappresenta un evidente aspetto del nursing in cui viene posta una grande enfasi sull'acquisizione delle competenze tecniche, mentre poco spazio è dato alla ricerca del razionale che sta alla base dell'azione stessa.

La detersione è una componente essenziale per la gestione delle ferite. Tuttavia, ci sono ricerche limitate a sviluppare protocolli. ${ }^{7}$

Una buona detersione provvede ad allontanare la maggior parte della carica batterica. Può essere consigliato utilizzare detergenti con surfattante, qualora si notino segni di contaminazione critica del letto dell'ulcera oppure in presenza di tessuto necrotico con slough. ${ }^{1,5}$ L'applicazione dovrebbe essere sospesa se compaiono effetti collaterali, come il bruciore non tollerato dal paziente.

Lo scopo di questa revisione è quello di focalizzare l'attenzione nell'ambito della medicazione o meglio sulle metodiche di detersione delle ulcere che rappresenta una componente molto importante per la gestione delle ferite, nonostante però vi siano pochi studi utili per la pratica quotidiana. Infatti, gli studi pubblicati si occupano prevalentemente del tipo di medicazione lasciando poco spazio alle soluzioni e alle tecniche che vengono utilizzate per detergere le ferite. Inoltre, non esiste un accordo uniforme tra i clinici sulla tipologia di soluzione da usare e sulla tecnica da preferire, ne consegue che le linee di condotta scelte tendono a riflettere le preferenze individuali. Pertanto, l'obiettivo della revisione è di verificare gli effetti della detersione sulle ferite considerando sia la tecnica che le soluzioni utilizzate, valutando come questi influiscono sui tassi di guarigione e infezione.

\section{MATERIALI E METODI}

Per rispondere all'obiettivo di questa revisione di letteratura si è proceduto ad identificare le parole chiave, quali: Abrasion, Cleansing, Irrigate, Laceration, Rin, Ulcer, Wash, Wound Shower.

Tali parole chiave sono state individuate e ricercate separatamente attraverso una ricerca grezza e successivamente unite dall'operatore booleano AND.
La stringa di ricerca usata è stata: "wound" OR "wounds" OR "bite" OR "ulcer" OR "ulcers" OR "abrasion" OR "abrasions" OR "laceration", AND: clean* OR wash* OR irrigation* OR shower* OR rins.

La ricerca della letteratura è avvenuta nelle banche dati di Pubmed e Cochrane Library dal 1 gennaio 2003 al 31 dicembre 2018. Sono stati considerati gli studi che rispettavano i seguenti criteri di inclusione: i) tipologie di studio: Meta-Analisi, Revisioni sistematiche, Trial Clinici Randomizzati; ii) popolazione di riferimento: popolazione adulta, né pediatrica, né anziana; iii) popolazione sana, dove l'ulcera rappresentava la patologia primaria e non secondaria ad altre patologie; iv) le parole chiave erano contenute all'interno del titolo e del riassunto; v) la lingua di stesura del lavoro era l'inglese.

Inoltre, sono stati esclusi gli studi con popolazioni rappresentate da pazienti con patologie psichiatriche ed oncologiche, pazienti con ustioni, detersione preintervento chirurgico e detersione con metodo Vac.

L'intervento ricercato negli studi è stato la detersione delle ferite ulcerative e l'outcome valutato è stata la riduzione del volume della ferita o l'aumento del tasso di guarigione delle ferite o la riduzione del tasso d'infezione.

\section{RISULTATI}

La ricerca bibliografica iniziale nelle banche dati di PubMed e Cochrane Library ha permesso di identificare 276 articoli rilevanti per l'obiettivo della nostra revisione. È stato successivamente eseguito uno screening dei titoli e dei riassunti e di questi sono stati considerati eleggibili per il nostro lavoro appena 15 articoli in versione integrale. Complessivamente, sono stati inclusi nella revisione finale soltanto 6 lavori. Nove articoli sono stati esclusi poiché non rispondevano ai criteri di inclusione ed allo scopo della nostra revisione. Tutti gli studi inclusi sono stati riassunti in Appendice. ${ }^{8-34}$

\section{DISCUSSIONE}

In questa revisione si è cercato di riassumere le evidenze attuali disponibili sulla detersione delle lesioni, evidenziando che non vi è alcuna prova che supporti l'uso di una particolare soluzione o tecnica di detersione. Inoltre i risultati ottenuti dovrebbero essere interpretati con cautela, in quanto ogni studio dovrebbe considerare la natura delle ferite, la qualità e la temperatura dell'acqua e la presenza di comorbilità dei pazienti selezionati. Alcuni degli studi inclusi presentano dei difetti metodologici nella fase di randomizzazione dei componenti dei campioni relativamente alle caratteristiche demografiche, alla grandezza dei campioni che solitamente sono piccoli e sottodimensionati. Inoltre, 
gli outcome come il comfort, la soddisfazione dei pazienti e quella degli operatori non sono sufficientemente descritti e necessitano di essere approfonditi.

Nello specifico la letteratura revisionata propone diverse soluzioni per la detersione delle lesioni ulcerative, quali: i) Acqua potabile di rubinetto: raccomandata con il vantaggio di essere efficiente, con un buon rapporto costoefficacia ed accessibile. ${ }^{14-17}$ Tuttavia, gli studi considerati ne sconsigliano l'utilizzo nella detersione delle ferite con esposizione ossea o tendini. In questi casi è raccomandato l'utilizzo si soluzione fisiologica $0.9 \%$ sterile; ii) Soluzione fisiologica: è la soluzione preferita per la detersione delle ferite perché è isotonica e non interferisce con il normale processo di guarigione, non danneggia i tessuti, non causa sensibilizzazione e non altera la normale flora batterica della cute. ${ }^{9-11}$ iii) Soluzione con poliesanide (PHMB) e betaina (Prontosan): è una soluzione d'irrigazione trasparente incolore contenente betaina, idrossido di sodio e acqua purificata. La soluzione è usata per detergere le ferite, per inumidire e lubrificare le medicazioni assorbenti per ulcere, ustioni, ferite post-chirurgiche e abrasioni. ${ }^{10} \mathrm{La}$ betaina è un tensioattivo efficace e particolarmente ben tollerato che disgrega il biofilm, scioglie le patine riducendo così la contaminazione della ferita da parte di batteri e detriti cellulari. La poliesanide (PHMB) è una sostanza antimicrobica efficace e straordinariamente ben tollerata che inibisce la crescita dei microrganismi e riduce la patina.

La letteratura propone le caratteristiche di una soluzione detergente una ferita come una sostanza non tossica per i tessuti umani, efficace in presenza di materiale organico, in grado di ridurre il numero di microrganismi, insensibile, ampiamente disponibile, con un buon rapporto costoefficacia, stabile. ${ }^{26}$ Tuttavia, Lawrence ${ }^{35}$ sostiene che qualsiasi effetto anti-microbico di irrigazione sia probabilmente dovuto all'azione fisica del fluido piuttosto che ai suoi eventuali effetti antimicrobici. Infatti, la maggior parte degli antisettici richiedono di essere in contatto con $\mathrm{i}$ batteri all'interno della ferita per più tempo di quello consentito per l'irrigazione.

È stato ancora suggerito che l'efficacia della detersione sia d'attribuire all'azione fisica vera e propria di pulizia grazie alla quale il materiale indesiderabile viene allontanato e rimosso dal letto della ferita piuttosto che al tipo di soluzione utilizzata. Per questo motivo, l'obiettivo delle diverse tecniche di detersione dovrebbe essere l'induzione di una forza idraulica adeguata in grado di vincere le forze adesive che mantengono batteri e contaminanti attaccati alla superficie della ferita. ${ }^{36}$ Tra gli antisettici disponibili la letteratura revisionata propone: i) Ipoclorito di sodio: utilizzato classicamente nelle ulcere da pressione con tessuto necrotico per ridurre l'incidenza delle infezioni. L'ipoclorito di sodio ha un effetto battericida contro la maggior parte degli organismi che si trovano comunemente nelle lesioni. É saltuariamente utilizzato su crescite cancerose per controllare la crescita batterica e ridurre al minimo l'odore. Tuttavia la soluzione è nota per il suo potere citotossico nei confronti delle cellule sane e dei tessuti di granulazione. Infine, il suo uso non è raccomandato per periodi superiori a 7-10 giorni; ii) Clorexidina: è un antisettico utilizzato ampiamente nel lavaggio delle mani e prodotti per via orale ma anche come disinfettante. ${ }^{37}$ Viene prodotto in due forme, ovvero allo $0.05 \%$ per la pulizia delle ferite ed al $4 \%$ per la preparazione dell'epidermide prima di un intervento chirurgico. Possiede un alto livello di attività antimicrobica, bassa tossicità e forte affinità per il legame con l'epitelio e le mucose. È più efficace contro i batteri gram positivi che gram negativi rispetto ai funghi ed ai bacilli tubercolari. É inattiva contro le spore di batteri, tranne che ad elevata temperatura; ${ }^{38}$ iii) Iodiopovidone (povidone iodio al 10\%): contiene polivinilpinzolidone iodio, solubile in acqua. Ha azione battericida ed è efficace contro una vasta gamma di batteri, funghi e spore. Soluzioni di iodio non sono efficaci in presenza di materiale organico, pus, slough e tessuto necrotico nelle ulcere, ${ }^{39}$ iv) Acqua ossigenata o perossido d'idrogeno: comunemente usata per l'antisepsi delle ulcere. Tuttavia alcuni studi riportano la sua efficacia nella guarigione delle ferite come antisettico, ma il suo utilizzo resta controverso. Alcuni studi hanno dimostrato la sua citotossicità per le cellule sane ed il tessuto di granulazione. Altri studi su animali e uomini non hanno dimostrato alcun effetto negativo sulla guarigione delle ferite. ${ }^{40}$ Subito dopo l'utilizzo di perossido di idrogeno è raccomandata l'irrigazione con soluzione fisiologica.

In letteratura una soluzione detergente viene definita come una sostanza non tossica per i tessuti umani, efficace in presenza di materiale organico, in grado di ridurre il numero di microrganismi, insensibile, ampiamente disponibile, con un buon rapporto costo-efficacia, stabile. ${ }^{40}$

Tuttavia si sostiene che qualsiasi effetto antimicrobico di irrigazione sia probabilmente dovuto all'azione fisica del fluido piuttosto che ai suoi eventuali effetti antimicrobici. Infatti, la maggior parte degli antisettici richiedono di essere in contatto con i batteri all'interno della ferita per più tempo di quello consentito per l'irrigazione. ${ }^{41} \mathrm{E}$ stato inoltre suggerito che l'efficacia della detersione sia da attribuire all'azione fisica vera e propria di pulizia grazie alla quale il materiale indesiderabile viene allontanato e rimosso dal letto della ferita piuttosto che dal tipo di soluzione utilizzata. Per questo motivo l'obiettivo delle diverse metodologie di detersione dovrebbero promuovere l'induzione di una forza idraulica adeguata in grado di vincere le forze adesive che mantengono batteri e contaminanti attaccati alla superficie della ferita. ${ }^{36}$

Dalla letteratura a disposizione emerge che le tecniche di detersione più comunemente utilizzate sono: i) 
l'irrigazione: eseguibile con una varietà di ausili come siringhe, perette, spray che hanno lo scopo d'immettere la soluzione detergente o l'acqua con una pressione sufficiente per rimuovere detriti cellulari, batteri e residui di medicazioni. Il modo più semplice per ottenere una adeguata pressione di lavaggio è utilizzare una siringa da $30 \mathrm{ml}$ con un ago da 18-20 gauge. In questo caso, oltre alle precauzioni per l'ago, occorre prestare attenzione a non esercitare troppa pressione perché questa può essere lesiva per il tessuto e perché l'operatore può essere investito dallo spruzzo di ritorno. I contenitori spray con erogatore manuale o sotto pressione sono più pratici anche se più costosi. In generale quando si utilizza la detersione per irrigazione occorre che le norme igieniche, per la prevenzione della contaminazione del personale e dell'ambiente, vengano scrupolosamente rispettate. ${ }^{29}$ Esistono molte altre procedure d'irrigazione della lesione come ad esempio la siringa a bulbo, il lavaggio ad alta pressione, dispositivi a lavaggio pulsatile ma sono costosi, ingombranti e difficile da mantenere sterili. L'irrigazione deve bilanciare da una parte l'effetto di pulizia e dall'altra il possibile trauma ai tessuti secondario ad un livello di pressione troppo alto: si parla pertanto di pressione d'irrigazione sicura e efficace. ${ }^{40}$ L'irrigazione della ferita rappresenta un' attività importante volta a ridurre il rischio di infezione della ferita, tuttavia non esistono evidenze ufficiali e raccomandazioni che ne descrivono la modalità di esecuzione in termini di metodi di irrigazione, soluzioni e volumi di detergenti da utilizzare; ii) l'immersione: tecnica dispendiosa ma efficace. Il paziente viene immerso con la parte interessata (più spesso gli arti inferiori) in vasche speciali dove l'acqua, talvolta addizionata ad antisettici, viene agitata con movimento forzato in modo da facilitare la rimozione del tessuto devitalizzato e reso meno aderente dall'uso di medicazioni occlusive; ${ }^{41}$ iii) il tamponamento: più frequentemente utilizzata poiché è la più rapida. S'impiegano di solito garze o tamponi di cotone imbevuti della soluzione e la detersione avviene per tamponatura o strofinamento dell'ulcera. In questo metodo la rimozione dei detriti e residui di medicazione avviene, più che altro in modo meccanico; occorre badare quindi a non ledere i tessuti. ${ }^{1}$

Un riguardo particolare dovrebbe essere rivolto alla temperatura della soluzione di lavaggio. Infatti, per non causare danni all'attività mitotica delle cellule, la temperatura del lavaggio dovrebbe essere compresa tra 28 e 30 gradi centigradi. In uno studio di Myers del 1982 si è visto che dopo un bagno a temperatura inferiore a 28 gradi centigradi occorrono $40 \mathrm{~min}$ per il recupero della temperatura iniziale e la divisione cellulare è bloccata per almeno 3 ore. Inoltre l'applicazione di soluzione detergente fredda può provocare una vasocostrizione, con diminuzione dell'apporto nutrizionale al tessuto, oltre che alla comparsa di sensazione dolorosa. ${ }^{42}$
A tal proposito Barnes et al. ${ }^{43}$ si sono proposti di ricercare le raccomandazioni attualmente disponibili per tale tematica con lo scopo di uniformare la pratica dell'irrigazione della ferita. A tal proposito l'American College of Surgeons definisce come alta la pressione di irrigazione una pressione compresa tra 15 e 35 psi, bassa pressione se compresa tra 1 e 15 psi. Numerosi studi sono stati eseguiti per valutare la pressione ottimale necessaria per l'irrigazione della ferita, e la maggior parti di essi ha mostrato che l'irrigazione ad elevata pressione risulta più efficace nella rimozione di batteri dal letto della ferita. Tuttavia, l'alta pressione di irrigazione è stata anche associata ad un maggior rischio di danneggiamento del tessuto e di propagazione dei batteri più in profondità: per questo dovrebbe essere evitata nelle lesioni in cui in rischio di contaminazione sia elevato e sia prevedibile la difficoltà nella rimozione dei batteri.

Non esistono in letteratura riferimenti sui valori di pressione di irrigazione ottimale. Ad ogni modo, la letteratura consiglia l'utilizzo di una pressione tra gli $8 \mathrm{e}$ 12 psi nelle ferite traumatiche per superare la forza adesiva dei batteri.

Non esistono, invece, raccomandazioni circa l'utilizzo di una modalità di irrigazione pulsatile o continua ed in termini di volume di detergente da utilizzare per un'irrigazione ottimale della ferita.

Per quanto riguarda i fluidi di detersione, la soluzione fisiologica rappresenta il detergente più comunemente utilizzato. Ciò nonostante numerosi detergenti, comunemente raggruppati nelle categorie di antibiotici, surfactante ed antisettici, sono stati combinati con i fluidi di irrigazione allo scopo di ottimizzare la prevenzione delle infezioni delle ferite.

Gli antibiotici risultano molto frequentemente utilizzati come additivi al fluido di irrigazione nonostante la carenza di evidenze che ne sostengono l'utilizzo ed i numerosi studi che suggeriscono il loro utilizzo con conseguenze deleterie favorendo al tempo stesso la resistenza batterica.

I surfactanti sono additivi progettati per favorire la rimozione dei batteri dalla ferita ma interferiscono con la loro abilità nell'aderire alle superfici.

Gli antisettici esercitano il loro potere battericida danneggiando la cellula batterica e la membrana. Lo iodio povidone è l'antisettico più comunemente utilizzato nonostante le scarse evidenze che ne sostengano l'efficacia. Inoltre, risulta tossico per le cellule tessutali, ritardando ed indebolendo la guarigione della ferita, particolarmente se utilizzato ad elevate concentrazioni.

Alcuni studi hanno dimostrato che lo iodo povidone, come altri disinfettanti, può essere diluito sufficientemente da mitigare il suo effetto tossico senza eliminare il suo potere battericida.

Barnes et al. ${ }^{43}$ sottolineano che l'unico antisettico 
attualmente approvato dalla Food and Drug Administration (FDA) per lo sbrigliamento e la pulizia delle ferite è un fluido d'irrigazione contenente acqua sterile e $0.05 \%$ di Clorexidina Gluconato $(\mathrm{CHG})$.

Gli autori concludono che l'irrigazione della ferita gioca un ruolo chiave non solo per ridurre il rischio di infezione della ferita, ma anche per ridurre la resistenza batterica, evitando la necessità di un trattamento post infezione monto più aggressivo (es. rimozione di impianti, inserimento di linee venose centrali e periferiche per l'infusione di antibiotici per via sistemica, ecc.), contenendone anche i costi per l'azienda sanitaria.

Infine gli autori consigliano di approfondire il tema di prevenzione delle infezioni allo scopo di standardizzare il tipo di soluzione d'irrigazione, il volume e il metodo da utilizzare per un'irrigazione ottimale del letto della ferita.

Gli autori concludono che esistono sufficienti evidenze che non raccomandano le soluzioni antibiotiche per l'irrigazione delle ferite e che l'opinione degli esperti potrebbe invece essere usata per guidare le migliori pratiche.

La gestione delle ulcere coinvolge molte figure professionali che devono collaborare per raggiungere degli obiettivi nell'ambito della guarigione delle ferite, ma spesso le ulcere vengono considerate come patologia a sé e non come una manifestazione secondaria ad una patologia di cui ne è segno o sintomo.

Inoltre essendo operatori specializzati in ambito di urgenze ci ritroviamo in difficoltà nella gestione delle ulcere in quanto c'è una carenza di formazione rispetto al wound care. Specificamente la detersione viene considerata un intervento di routine che viene effettuato con soluzione fisiologica sterile attraverso il metodo per tamponamento, questo perché la letteratura non fornisce ricerche soddisfacenti nell'ambito dell'utilizzo dell'acqua di rubinetto.

Inoltre nelle consuetudini di reparto la soluzione fisiologica è considerata come unico detergente esistente, anche se non è determinato da un razionale scientifico ma per lo più da ragioni pratiche e dalle consuetudini degli operatori.

Dalla nostra revisione è emerso che la soluzione fisiologica è, con tutta probabilità, l'agente detergente più applicato nel mondo, è isotonica con i fluidi della ferita e per tale ragione non cede né sottrae liquido alla stessa; questa sua caratteristica gli permette da un lato di esercitare appieno l'azione meccanica di pulizia della ferita ma dall'altro ne fa un prodotto costoso e di gestione prevalentemente ospedaliera.

Lo studio clinico randomizzato di Griffiths et al. ${ }^{13}$ ha confrontato gli effetti dell'acqua di rubinetto e della normale soluzione salina sui tassi di guarigione e di infezione delle ferite acute e croniche. Il processo è stato condotto in due centri sanitari della comunità metropolitana nel Nuovo Galles del Sud, in Australia. Trentacinque pazienti con 49 ferite acute o croniche sono stati randomizzati a ricevere l'irrigazione della ferita con soluzione fisiologica o acqua di rubinetto. L'analisi statistica ha dimostrato che non vi era alcuna differenza significativa tra l'infezione ed i tassi di guarigione nelle ferite irrigate con soluzione fisiologica o acqua di rubinetto. Sebbene la piccola dimensione del campione sia una limitazione di questo studio, i ricercatori concludono che l'acqua potabile del rubinetto sembra fornire un'alternativa sicura alla normale soluzione salina per la pulizia delle ferite e può essere preferita da alcuni pazienti.

Tuttavia la decisione di usare l'acqua di rubinetto deve tener conto della qualità dell'acqua, della natura della lesione e delle condizioni generali del paziente. Inoltre, secondo gli autori negli studi futuri si dovrebbe uniformare le caratteristiche dei partecipanti, dell'acqua, la durata del processo di detersione, il volume del liquido del detergente utilizzato, come anche gli strumenti e il metodo di detersione. In assenza di acqua potabile di rubinetto l'acqua bollita raffreddata o l'acqua distillata possono essere usati come detergenti delle ferite.

Nello studio di Moore et al. ${ }^{20}$ invece vengono confrontate la soluzione contenente aloe vera e la soluzione fisiologica. Il trattamento con soluzione contenente aloe vera ha prodotto risultati statisticamente significativi rispetto al trattamento con soluzione fisiologica o acqua di rubinetto. Inoltre, per quanto riguarda la tecnica, le ulcere da pressione deterse con lavaggio pulsatile hanno riportato una riduzione statisticamente significativa del volume dell'ulcera rispetto al gruppo di simulazione.

Nello studio di Romanelli et al. ${ }^{24}$ il $\mathrm{pH}$ di superficie delle ulcere venose trattate con prontosan è stato confrontato col $\mathrm{pH}$ di superficie delle ulcere venose trattate con soluzione fisiologica. Le lesioni deterse con il prontosan riportavano un significativo miglioramento nel controllo della carica batterica e nella riduzione del $\mathrm{pH}$ della superficie della lesione.

Tuttavia il campione selezionato era di piccole dimensioni e non è stato possibile rendere ben evidente il miglioramento oggettivo del tasso di guarigione delle ferite. A tal proposito gli autori concludono che la dimensione delle ferite per entrambi i campioni di trattamento è rimasta invariata, rimandando a studi futuri la valutazione del $\mathrm{pH}$ associato ad altri indicatori di valutazione delle ferite ulcerative.

Nello studio di Moscati et al. ${ }^{17} \mathrm{i}$ tassi d'infezione di ferite semplici, che necessitano di sutura, irrigate con acqua di rubinetto o con soluzione fisiologica all'interno di un dipartimento d'emergenza sono stati confrontati tra loro riportando lo stesso tasso d'infezione in entrambi i gruppi di trattamento. Rispetto alla soluzione fisiologica, 
l'acqua di rubinetto per l'irrigazione delle ferite sembra essere più conveniente ed altrettanto sicura ed efficace, rivalutandola soprattutto nei contesti dei dipartimenti d'emergenza come una valida alternativa alla soluzione fisiologica.

Tuttavia i dati raccolti nello studio dovrebbero essere considerati con attenzione, poiché non sono state descritte le eventuali indicazioni nella gestione della ferita ulcerativa con acqua di rubinetto a domicilio. Inoltre, la somministrazione del questionario telefonico per la valutazione degli indicatori di infezione non risulta essere lo strumento più idoneo per misurare gli outcomes dello studio.

Lo studio di Mak et al. ${ }^{25}$ confronta il metodo di detersione dell'irrigazione a pressione con quello di swabbing nelle ferite che guariscono per seconda intenzione. Gli autori hanno dimostrato che l'irrigazione a pressione applicata alle ferite che guariscono per seconda intenzione è più sicura, più conveniente e riduce $\mathrm{i}$ tempi di guarigione delle ferite. Tuttavia i campioni ottenuti risultavano non omogenei e poco equilibrati a causa delle differenti eziologie. Per quanto riguarda il dolore è stata segnalata una maggiore soddisfazione nei pazienti del gruppo trattato con l'irrigazione pressurizzata. Non è stata riportata alcuna differenza clinicamente importante dei tassi d'infezione della ferita tra i due gruppi.

Il New South Wales Center for Evidence Based Nursing \& Midwifery in collaborazione con il Joanna Briggs Institute hanno condotto una revisione sistematica ${ }^{26}$ riportando l'efficacia delle soluzioni, nonché la tecnica di detersione e pressione da applicare nella detersione delle ferite. Tutto ciò ha evidenziato che l'acqua di rubinetto è raccomandata solo se potabile nella detersione di lacerazioni, ferite chirurgiche suturate, ed in caso di ferite croniche. In aggiunta si raccomanda l'acqua bollita e raffreddata come una valida alternativa per detergere le ferite. Inoltre, la revisione ha sottolineato come fare la doccia bagnando la ferita chirurgica non aumenta il rischio d'infezione e neppure rallenta il processo di guarigione ma ne favorisce il benessere del paziente. Per quanto riguardo l'utilizzo dello iodio povidone, quest'ultimo può essere usato per detergere ferite contaminate ma successivamente se ne consiglia il risciacquo.

La revisione raccomanda di esercitare pressioni durante la detersione di 13 psi, ritenute efficaci nel ridurre l'infezione in caso di ferite e lacerazioni traumatiche.

Lo studio condotto Barnes et al. ${ }^{43}$ riporta che la pressione ottimale necessaria per la detersione delle ferite acute è l'irrigazione ad elevata pressione. Tuttavia, l'alta pressione di irrigazione è stata anche associata ad un maggior rischio di danneggiamento del tessuto e di propagazione dei batteri più in profondità: per questo dovrebbe essere evitata nelle lesioni in cui in rischio di contaminazione sia elevato e sia prevedibile la difficoltà nella rimozione dei batteri.

Lo studio di Khan et al. ${ }^{27}$ ha riportato informazioni circa l'utilizzo di antisettici, soprattutto di iodio povidone che, nella gestione delle ferite acute, è rimasta una sostanza inibitoria per la crescita e lo sviluppo di microorganismi patogeni.

Lo iodio povidone contiene polivinilpirrolidone che è un idrosolubile complesso di iodio in soluzione al $10 \%$ con acqua. Ha un'azione battericida ed efficacia contro una vasta gamma di batteri, funghi e persino spore. La sua azione battericida si esplica in pochi secondi mediante l'inattivazione dei substrati citoplasmatici, che sono necessari per la vita dei batteri. La presenza di materia organica ha un effetto depressivo sulle concentrazioni di iodio. In assenza d'inibitori della disinfezione agisce in 10 secondi.

Tuttavia nello studio di Robert et al. ${ }^{44}$ non sono stati riportati effetti negativi dello iodio povidone ed il tasso d'infezione delle lesioni trattate con quest'ultimo era più basso rispetto al gruppo di controllo.

\section{CONCLUSIONI}

La tecnica e le soluzioni detergenti utilizzate per la detersione delle ferite rimangono un dibattito aperto e irrisolto. La routine della detersione delle ferite varia da paese a paese, ospedali e dipartimenti, e molto spesso sia la scelta del liquido di detersione che la tecnica di detersione da utilizzare non è motivata da un reale razionale scientifico ma esclusivamente da ragioni pratiche e dall'abitudine degli operatori.

Infatti, non esistono linee guida riguardo un valore di pressione di irrigazione ottimale, tuttavia la letteratura consiglia l'utilizzo di una pressione che varia tra gli 8 ed i 12 psi nelle ferite traumatiche per superare la forza adesiva dei batteri.

Ed ancora, non esistono raccomandazione per quanto riguarda l'utilizzo di una modalità di irrigazione pulsatile o continua ed in termini di volume di detergente da utilizzare per un'irrigazione ottimale della ferita.

Per quanto riguarda i fluidi di detersione, la soluzione fisiologica rappresenta il detergente più comunemente utilizzato. Ciò nonostante numerosi detergenti, comunemente raggruppati nelle categorie di antibiotici, surfactante ed antisettici, sono stati combinati con i fluidi di irrigazione allo scopo di ottimizzare la prevenzione delle infezioni delle ferite.

Si sostiene che la detersione debba comprendere sia la tecnica, sia la soluzione che gli strumenti, tuttavia la letteratura scientifica di settore offre attualmente scarsa evidenza per comprendere con chiarezza quale sia il tipo di soluzione e la tecnica di detersione più idonei, né esiste 
una consolidata base di consenso scientifico sui criteri di impiego dei fluidi.

L'agente detergente ideale dovrebbe non essere tossico né allergogeno, restare attivo in presenza di materiale organico, essere in grado di diluire la carica microbica, economico e di facile stoccaggio.

Tuttavia la presenza contemporanea di tutti questi caratteri è particolarmente difficile da reperire in un unico fluido.

Secondo le indicazioni emerse dalla letteratura studiata, l'implementazione della detersione nella gestione delle ferite deve innanzitutto prevedere un progetto di formazione rivolto a tutto il team multiprofessionale, il quale sviluppo deve essere supportato non solo dal singolo professionista ma anche dalla politica organizzativa. In particolare il programma di implementazione dovrà: i) prevedere un evento formativo con presentazione di contenuti didattici e multimediali che contrappongono la detersione con soluzione fisiologica attualmente in uso nell'unità operativa con acqua di rubinetto e prontosan proposte dalla letteratura; ii) fornire indicazioni su come ottenere le informazioni cruciali in ambito della tecnica da utilizzare contrapponendo la tecnica di tamponamento, alla tecnica di irrigazione pressurizzata con siringa da 30 $\mathrm{ml}$ e ago cannula 18 gauge (metodo d'irrigazione più economico disponibile in reparto per garantire una pressione maggiore ad $8 \mathrm{psi}$ ); iii) fornire materiale didattico di consultazione prontamente disponibile alla consultazione; vi) promuovere l'utilizzo della scheda di valutazione delle lesioni informatizzata da poco introdotta nel reparto rendendo disponibile delle postazioni computer posizionate su carrelli, permettendo una maggiore accessibilità alle informazione del paziente e allo strumento elettronico.

Infine, sarà importante accompagnare i professionisti nell'applicazione della nuova tecnica di detersione ed all'utilizzo dei nuovi detergenti durante tutto il percorso formativo. A tale scopo risulta importante individuare un infermiere specializzato in wound care in modo da fornire a tutti i membri del team un supporto per affrontare le eventuali situazioni di criticità.

Una volta implementate le nuove tecniche di detersione, sarebbe interessante osservare se effettivamente queste vengano utilizzate con continuità nella pratica quotidiana e se tale applicazione determini un miglioramento nel processo di guarigione delle ferite, della continuità assistenziale nella gestione delle ferite, nonché un aumento della soddisfazione dei pazienti e del personale sanitario. Per fare questo si dovrebbe organizzare una raccolta dati prima e dopo l'introduzione dei nuovi detergenti e delle nuove tecniche di detersione.

In conclusione, per funzionare tutto ciò richiede lo sforzo e la collaborazione di tutto lo staff. ${ }^{45}$

\section{BIBLIOGRAFIA}

1. Monti M (ed). L'ulcera cutanea - Approccio multidisciplinare alla diagnosi ed al trattamento. Milano: Springer Verlag; 2000.

2. Vowden K. VPB. Bridging the gap: the impact of patient choice on wound care. J Wound Care 2006;15:143-5.

3. Lindholm C, Bergsten A, Berglund E. Chronic wounds and nursing care. J Wound Care 1999;8:5-10.

4. Ricci E, Cassino R. Piaghe da decubito. II ed. Torino: Minerva Medica; 2004.

5. Dowsett C, Claxton K. Reviewing the evidence for wound bed preparation. J Wound Care 2006;15:439-42.

6. Preece J. Development of a wound-management formulary for use in clinical practice Prof Nurse 2004;20:27-9.

7. Lindholm C, Bergsten A, Berglund E. Chronic wounds and nursing care. J Wound Care 1999;8:5-10.

8. Fernandez R, Griffiths R. Water for wound cleansing. Cochrane Database Syst Rev 2008;1:CD003861. doi:10.1002/14651858.CD003861.pub2

9. Goldberg H, Rosenthal S, Nemetz J. Effect of washing closed head and neck wounds on wound healing and infection. Am J Surg 1981;141:358-9.

10. Riederer SR, Inderbitzi R. Does a shower put postoperative healing at risk? Der Chirurg 1997;68:715-7.

11. Neues C, Haas P. Modification of postoperative woundhealing by showering [Beeinflussung der postoperativen Wundheilung durch duschen]. Der Chirurg 2000;71:234-6.

12. Angeras MH, Brandberg A, Falk A, Seeman T. Comparison between sterile saline and tap water for the cleaning of acute traumatic soft tissue wounds. Eur J Surg 1992;158:347-50.

13. Griffiths RD, Fernandez RS, Ussia CA. Is tap water a safe alternative to normal saline for wound irrigation in the community setting? J Wound Care 2001;10:407-11.

14. Bansal BC, Wiebe RA, Perkins SD, Abramo TJ. Tap waterfor irrigation of lacerations. Am J Emerg Med 2002;20:469-72.

15. Godinez FS, Grant-Levy TR, McGuirk TD, et al. Comparison of normal saline vs tap water for irrigation of minor lacerations in the emergency department. Acad Emerg Med 2002;19:396-7.

16. Valente JH, Forti RJ, Freundlich LF, et al. Wound irrigation in children: saline solution or tapwater? Ann Emerg Med 2003;41:609-16.

17. Moscati RM, Mayrose J, Reardon RF, et al. A multicentre comparison of tap water versus sterile saline for wound irrigation. Acad Emerg Med 2007;14:404-10.

18. Museru LM, Kumar A, Ickler P. Comparison of isotonic saline, distilled water and boiled water in irrigation of open fractures. Int Orthop1989;13:179-80.

19. Tay SK. Is routine procaine spirit application necessary in the care of episiotomy wound? Singapore Med J 1999;40:581-3.

20. Moore ZE, Cowman S. Wound cleansing for pressure ulcers. Cochrane Database Syst Rev 2013;3:CD004983. doi:10.1002/14651858.CD004983.

21. Bellingeri A, Forma O, Polignano R, Attolini R, Accardi S, Fabbri $\mathrm{C}$, et al. Multi-centre research on a cleanser for cutaneous wounds. Wound Repair and Regeneration 
2003;11:A36. In: Moore ZEH, Cowman S. Wond cleasing for pressure ulcers. Cochrane Database of Systematic Reviews 2005;4:108. doi: 10.1002/14651858.CD004983.pub2

22. Van Der Cammen TJM, O'Callaghan U, Whitefield M. Prevention of pressure sores, a comparison of new and old pressure sore treatments. Br J Clin Pract 1987;41:1009-11.

23. Hartman D, Coetzee JC. Two US practitioners' experience of using essential oils for wound care. J Wound Care 2002;11:317-20.

24. Romanelli M, Dini V, Barbanera S, et al. Evaluation of the efficacy and tolerability of a solution containing propyl betaine and polihexanide for wound irrigation. Skin Pharmacol Physiol 2010;23:41-4. doi: 10.1159/000318266.

25. Mak SS, Lee MY, Cheung JS, et al. Pressurised irrigation versus swabbing method in cleasing wounds healed by secondary intention: a randomised controlled trial with costeffectiveness analysis. Int J Nurs Stud 2015;52:88-101. doi: 10.1016/j.ijnurstu.2014.08.005.

26. Joanna Briggs Institute. Solutions, techniques and pressure in wound cleasing. Nurs Stand 2008;22:35-9.

27. Khan MN, Davies CG. Advances in the management of leg ulcers - the potential role of growth factor. Int Wound J 2006;3:113-22. doi: 10.1111/j.1742-4801.200211.x

28. Longmire JL, Albright KL, Lewis AK, et al. A rapid and simple method for the isolation of high molecular weight cellular and chromosome-specific DNA in solution without the use of organic solvents. Nucleic Acids Res 1987;15:859. doi:10.1093/nar/15.2.859

29. Chisholm CD, Cordell WH, Rogers K, Woods JR. Comparison of a new pressurized saline canister versus syringe irrigation for laceration cleansing in the emergency department. Ann Emerg Med 1992;2:1364-7.

30. Morse J, Miles M, Clark D, Doberneck B. 'Sensing' patient needs: Exploring concepts of nursing insight and receptivity used in nursing assessment. Sch Inq Nur Pract 1994;8:233-55.

31. Weller K. In search of efficacy and efficiency. An alternative to conventional wound cleansing modalities. Ostomy Wound Manage 1991;37:23-8.

32. Hollander JE, Valentine SM, McCuskey CF, et al. The Stony Brook Wound Registry Study Group. Long-term evaluation of cosmetic appearance of repaired lacerations: validation of telephone assessment. Ann Emerg Med 1998;31:92-8.

33. Fraser I, Askew A, Biles J, Pinchin J. Prospective randomized controlled trial of early postoperative bathing. Br Med J 1976;1:1506-7.

34. Burke DT, Ho CH, Saucier MA, Stewart G. Effects of hydrotherapy on pressure ulcer healing. Am J Phys Med Rehabil 1998;77:394-8.

35. Lawrence JC. Wound irrigation. J Wound Care 1997;6:23-6.

36. Atiyeh BS, Dibo SA, Hayek SN. Wound claesing, topical antiseptics and wound healing. Int Wound J 2009;6:420-30. doi: 10.1111/j.1742-481X.2009.00639.x.

37. Thomas GW, Rael LT, Bar-Or RBS, et al. Mechanisms of delayed wound healing by commonly used antiseptics. J Trauma 2009;66:81-91. doi: 10.1097/TA.0b013e31818b146d.

38. Meijers JMM, Jos MGA, Pam AJMP, et al. Differences in nutritional care in pressure ulcer patients whether or not using nutritional guidelines. Nutrition 2008;24:127-32.

39. Rodeheaver GT. Pressure ulcer debridement and cleansing: A review of current literature. Ostomy Wound Manag 1999;45:80S-85S.

40. Flanagan M. Wound management. Edimburgh: Churchill Livingstone 1997.

41. Furlini S, Somà K. Lettura della ferita. Trattamento localemetodologia di approccio alla lcc: L'importanza di adottare un metodo di lavoro. Disponibile da: http://www. lesionicutaneecroniche.it/TRATTAMENTOLOCALE\%20C D/PDF/lettura.pdf

42. Myers JA. Modern plastic surgical dressing. Health Society Service Journal 1982;4:336-7. In: Grainger AN. Essential Practice for healthcare Assistants. Mark Allen Healthcare Limited; 2009.

43. Barnes S, Spencer M, Graham D, Jonhnson HB. Surgical wound irrigation: a call for evidence-based standardization of practice. Am J Infect Control 2014;42:525-9. doi: 10.1016/j.ajic.2014.01.012.

44. Robert IB. Povidone-Iodine Solution in Wound Treatment. Phys Ther 1998;78:212-8.

45. Vitale, E. Clinical teaching models for nursing practice: a review of literature. Prof Infer 2014;67:117-25. doi: 10.7429/pi.2014.672117. 